\title{
THE HOLOMORPHIC EXTENSION OF CR FUNCTIONS NEAR A POINT OF HIGHER TYPE
}

\author{
A. BOGGESS
}

(Communicated by Irwin Kra)

\begin{abstract}
Suppose $M$ is a submanifold of $C^{n}$ with real codimension at least one. A geometric description is given of the local hull of holomorphy of an open subset of $M$ which contains a point of higher type in which all Hormander numbers are the same. This result is proved as a consequence of examining the relationship between the hypoanalytic wave front sets of CR functions on $M$ and CR extension to a manifold of one higher dimension than $M$.
\end{abstract}

0. Introduction. Recently, Baouendi and Rothschild in [BR] have shown that on a semirigid submanifold of higher type that CR functions near a point $p$ holomorphically extend to an open wedge in $C^{n}$. In that paper, higher type means that all the Hormander numbers are finite. The purpose of this paper is to more carefully examine the case when all the Hormander numbers are the same finite number $l$ and to give a more precise description of this wedge under this additional assumption. We show (Theorem 1.4 below) that this wedge fills in the convex hull of the image of the $l$ th Levi form at $p$. This result is the natural generalization of the results in [BP and $\mathbf{B}$ ] which handle the cases $l=2$ and $l=3$ respectively.

There are two key components of the proof of this result. First, we use a CR extension result in [BPi] which states that CR extension to a submanifold of one higher dimension is always possible near a point $p$ of type $l(l<\infty)$. Secondly, we examine (in Lemma 1.7 below) the relationship between CR extension to a submanifold of one higher dimension and the hypoanalytic wave front sets of CR functions defined near $p$.

1. Notation and the precise statement of results. Throughout this paper $M$ will denote a smooth generic real submanifold of $C^{n}$ of real codimension $d$, $1 \leq d \leq n$ and $p$ will be a point in $M$. We let $T_{p}(M)$ be the real tangent space of $M$ at $p$ and we let $H_{p}(M)$ be the $J$-invariant subspace of $T_{p}(M)$, where $J: R^{2 n} \rightarrow R^{2 n}$ is the linear isometry induced by multiplication by $\sqrt{-1}$. The totally real tangent space of $M$ at $p$, denoted $T_{p}^{0}(M)$, is the orthogonal complement of $H_{p}(M)$ in $T_{p}(M)$ under the usual inner product on $R^{2 n}$. Since $M$ is generic, $\operatorname{dim}_{R} H_{p}(M)=2 m$ where $m=n-d$ and $\operatorname{dim}_{R} T_{p}^{0}(M)=d$. We let $N_{p}(M)$ (the normal space of $M$ ) be the orthogonal complement of $T_{p}(M)$ in $R^{2 n}$. The map $J$ is an isometry from $T_{p}^{0}(M)$ to $N_{p}(M)$.

Our description of hulls of holomorphy will involve wedges (or conoids) emanating from $M$ in the normal direction. Therefore to simplify this description, we shall

Received by the editors November 5, 1986 and, in revised form, July 13, 1987. Results presented at the AMS special session in Several Complex Variables at Indianapolis, Indiana, April, 1986.

1980 Mathematics Subject Classification (1985 Revision). Primary 32D15; Secondary 32 E99.

Research partially supported by NSF Grant \#MSC-8301369. 
assume that $p=0$ and that

$$
M=\left\{(z, w)=(x+i y, w) \in C^{d} \times C^{m} ; y=\phi(x, w)\right\}
$$

where $\phi: R^{d} \times C^{m} \rightarrow R^{d}$ is smooth with $\phi(0)=0$ and $D \phi(0)=0$. In these coordinates, we have

$$
\begin{aligned}
& T_{0}(M)=\left\{(x+i 0, w) ; x \in R^{d}, w \in C^{m}\right\} \\
& N_{0}(M)=\left\{(0+i y, 0) ; y \in R^{d}\right\} \\
& H_{0}(M)=\left\{(0, w) ; w \in C^{m}\right\} \\
& T_{0}^{0}(M)=\left\{(x+i 0,0) ; x \in R^{d}\right\}
\end{aligned}
$$

We let $Z: R^{d} \times C^{m} \rightarrow C^{d}$ be defined by

$$
Z(x, w)=x+i \phi(x, w) \text {. }
$$

Thus, a local parametrization of $M$ is given by the map $(x, w) \rightarrow(Z(x, w), w)$.

The open sets to which we will extend CR functions as holomorphic functions will be called conoids and are defined as follows.

DEFINITION 1.2. For a set $\omega \subset M$ and an open cone $\Gamma \subset N_{0}(M)$ and an open set $V$ in $C^{n}$ containing 0 we define the conoid

$$
N_{V}(\omega, \Gamma):=\left\{\left(Z(x, w)+i Z_{x}(x, w) \cdot \nu, w\right) \in V ; \nu \in \Gamma \text { and }(Z(x, w), w) \in \omega\right\}
$$

where $Z_{x}$ represents the derivative of $Z$ with respect to $x$ and $Z_{x}(x, w) \cdot \nu$ represents multiplication of the $d \times d$ matrix $Z_{x}(x, w)$ with the $d$-vector $\nu$. Since $Z_{x}(0,0)$ is the identity, $N_{V}(\omega, \Gamma)$ approximates $\omega+\Gamma$. Since one can always locally graph $M$ over its tangent space at a point $p \in M$, the above definition of the conoid can easily be extended to define $N_{V}(\omega, \Gamma)$ for an arbitrary submanifold which is not necessarily presented as in (1.1).

DEFINITION 1.3 [BCT]. For an open set $\omega \subset M$ and cone $\Gamma \subset T_{0}^{0}(M)$, we say that $f \in B_{V}(\omega, \Gamma)$ if $f(z, w)$ is holomorphic in $z$ for $(z, w) \in N_{V}(\omega, \Gamma)$ and:

To every compact set $K \subset \omega$ there is an integer $k \geq 0$ and a constant $C>0$ such that $|f(q)| \leq C(\operatorname{dist}(q, M))^{-k}$ for all $q \in N_{V}(K, \Gamma)$.

We shall assume that the open set $\omega \subset M$ is of the form

$$
\omega=\left\{(Z(x, w), w) ;|x|<r_{1},|w|<r_{2}\right\}
$$

for some $r_{1}, r_{2}>0$. For fixed $w \in C^{m}$ let

$$
\omega_{w}=\left\{(Z(x, w), w) ;|x|<r_{1}\right\} .
$$

Let $\Gamma \subset T_{0}^{0}(M)$ be an acute cone, i.e. a cone whose closure is properly contained in a half space of $T_{0}^{0}(M)$. As shown in [BCT, §II.1], if $f \in B_{V}(\omega, \Gamma)$ then the distributional boundary values of $f$ on $\omega_{w}$ exist (and will be denoted by $b_{w} f$ ) in the following sense: if $\psi \in C_{0}^{\infty}\left(\left\{x \in R^{d}:|x|<r_{1}\right\}\right)$ and if $\nu \in \Gamma$ then

$$
\left\langle b_{w} f, \psi\right\rangle:=\lim _{t \rightarrow 0^{+}} \int_{x \in R^{d}} f\left(Z(x, w)+i t Z_{x}(x, w) \cdot \nu, w\right) \psi(x) d_{x} Z(x, w)
$$

where

$$
d_{x} Z(x, w)=d_{x} Z_{1}(x, w) \wedge \cdots \wedge d_{x} Z_{d}(x, w)
$$


This limit is independent of the vector $\nu \in \Gamma$ as shown in [BCT, Lemma 1.5]. The boundary values of $f$ on $\omega$ (denoted $b f$ ) are defined analogously: if $\psi \in$ $C_{0}^{\infty}\left(\left\{(x, w) ;|x|<r_{1},|w|<r_{2}\right\}\right)$ then

$$
\langle b f, \psi\rangle:=\lim _{t \rightarrow 0^{+}} \iint_{x \in R^{d} w \in C^{m}} f\left(Z(x, w)+i t Z_{x}(x, w) \cdot \nu, w\right) \psi(x, w) d_{x, w} Z(x, w) d \lambda(w)
$$

where

$$
d_{x, w} Z(x, w)=d_{x, w} Z_{1}(x, w) \wedge \cdots \wedge d_{x, w} Z_{d}(x, w)
$$

and where $d \lambda(w)$ is the volume form defined on $C^{m}$. Of course, $b f$ and $b_{w} f$ as defined above are really distributions on $R^{d} \times C^{m}$ and $R^{d}$ respectively. However, one can use the diffeomorphisms $(x, w) \rightarrow(Z(x, w), w)$ and $x \rightarrow(Z(x, w), w)$ to transfer $b f$ and $b_{w} f$ to $\omega$ and $\omega_{w}$ respectively. Since $w$ is treated as a parameter, $f$ need only be holomorphic in $z$ for the above boundary values to make sense.

In order to give a description of the local hull of holomorphy, we need to define the Levi forms. We let $H_{p}^{C}(M)$ be the complexification of $H_{p}(M)$ for $p \in M$. Since $M$ is CR, $H^{C}(M)$ forms a subbundle of the complexified tangent bundle of $M$. We have $H^{C}(M)=H^{1,0}(M)+H^{0,1}(M)$ which gives the splitting of $H^{C}(M)$ into the $+i$ and $-i$ eigenspaces of $J$. We say that $p$ is a point of type $l(l \geq 2)$ if all Lie brackets of vector fields from $H^{C}(M)$ of length $l-1$ at $p$ are contained in $H_{p}^{C}(M)$ and there exists a Lie bracket of vector fields from $H_{p}^{C}(M)$ of length $l$ at $p$ which is not contained in $H_{p}^{C}(M)$. The length of a Lie bracket is the number of vector fields appearing in the bracket. For a point $p$ of type $l$, we define the $l$ th Levi form $L_{p}^{l}: H_{p}^{1,0}(M) \rightarrow T_{p}^{0}(M)$. If $X_{p} \in H_{p}^{1,0}(M)$, then

$$
L_{p}^{l}\left(X_{p}\right):=\frac{1}{2 i} \pi_{p}\left[\sum_{\varepsilon_{1}, \ldots, \varepsilon_{l-2}} C_{\varepsilon}\left[X^{\varepsilon_{1}},\left[X^{\varepsilon_{2}}, \ldots,\left[X^{\varepsilon_{l-2}},[X, \bar{X}]\right] \cdots\right]\right]_{p} .\right.
$$

Here $X \in H^{1,0}(M)$ is a vector field extension of $X_{p}$ and $\pi_{p}: T_{p}^{C}(M) \rightarrow T_{p}^{0}(M)$ is the orthogonal projection map. The sum is taken over all $\varepsilon=\left(\varepsilon_{1}, \ldots, \varepsilon_{l-2}\right)$ with $\varepsilon_{i}=+1$ or -1 with the convention that $X^{1}=X$ and $X^{-1}=\bar{X} \in H^{0,1}(M)$. Finally $C_{\varepsilon}=1 /(q+1) !(l-q-1)$ ! where $q$ is the number of occurrences of +1 in the set $\left\{\varepsilon_{1}, \ldots, \varepsilon_{l-2}\right\}$. This definition is independent of the choice of vector field extension $X \in H^{1,0}(M)$ of $X$. In [BPi, Lemma 5.2], a formula for $L_{p}^{l}$ is given in terms of the $l$ th order complex hessian of a set of defining functions for $M$. When $l=2$, this Levi form is just a constant multiple of the usual Levi form.

Finally, we say that the cone $\Gamma_{1} \subset N_{p}(M)$ is smaller than the cone $\Gamma_{2} \subset N_{p}(M)$ (and we write $\Gamma_{1}<\Gamma_{2}$ ) if $\Gamma_{1} \cap S \subset \subset \Gamma_{2} \cap S$ where $S$ is the unit sphere in $N_{p}(M)$ centered at $p$. Our main result on CR extension is contained in the following theorem.

THEOREM 1.4. Suppose $M$ is a smooth $C R$ generic submanifold of $C^{n}$ and $p$ is a point of type l in $M$ and $\omega$ is an open set in $M$ containing $p$. Let $\Gamma \subset T_{p}^{0}(M)$ be the convex hull of the image of the lth Levi form of $M$ at $p$. Suppose the interior of $\Gamma$ (with respect to the topology of $T_{p}^{0}(M)$ ) is nonempty. Then there exists an open 
set $\Omega$ in $C^{n}$ with the following properties:

(i) For each cone $\Gamma^{\prime} \subset N_{p}(M)$ which is smaller than $J\{\Gamma\}$ there is an open set $\omega^{\prime}$ in $M$ and an open set $V^{\prime}$ in $C^{n}$ with $p \in \omega^{\prime}$ and $p \in V^{\prime}$ and such that $N_{V}\left(\omega^{\prime}, \Gamma^{\prime}\right) \subset \Omega$.

(ii) For each $C R$ distribution $u$ defined on $\omega$ there is a holomorphic function $U$ defined on $\Omega$ with $U \in B_{V}\left(\omega^{\prime}, \Gamma^{\prime}\right)$ and with $b U=u$ on $\omega^{\prime}$.

The theorem conveys the following picture of $\Omega$ (in codimension two).

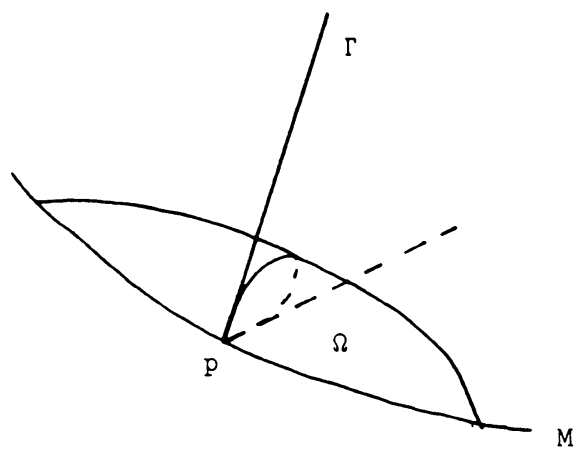

REMARK. Recall that since $\Gamma \subset T_{p}^{0}(M)$ clearly $J\{\Gamma\} \subset N_{p}(M)$. Since $\operatorname{dim}_{R} T_{p}^{0}(M)=d$, the hypotheses on $\Gamma$ guarantees that $\Gamma$ is a $d$-dimensional (i.e. maximal dimensional) cone. This is equivalent to saying that all the Hörmander numbers are the same finite number (namely $l$ ). The theorem essentially says that if $\Gamma$ is the convex hull of the image of the $l$ th Levi form, then one can locally CR extend in the directions given by $J\{\Gamma\}$. Note that the tangent cone of $\Omega$ at $p$ is spanned over $R^{+}$by $J\{\Gamma\}$ and $T_{p}(M)$.

As a first step in the direction of the proof of this theorem, we shall need the following theorem from [BPi] which roughly states that CR extension near a point of type $l$ to a manifold of one higher dimension is possible in the directions which are essentially in the image of the $l$ th Levi form.

THEOREM 1.5 (see [BPi], Theorem 1.1 and subsequent remarks). Let $M$ be a smooth generic $C R$ submanifold of $C^{n}$ and let $p \in M$ be a point of type $l \geq 2$. Let $\omega$ be an open set in $M$ with $p \in \omega$ and let $\nu \in T_{p}^{0}(M)$ be a unit vector in the image of $L_{p}^{l}$. Then for every $\varepsilon>0$ there is a unit vector $\nu_{\varepsilon} \in T_{p}^{0}$ and a smooth manifold $\widetilde{M}_{\varepsilon}$ with boundary such that the following hold:

(i) $\left\|\nu-\nu_{\varepsilon}\right\|<\varepsilon$.

(ii) $\operatorname{dim}_{R} \widetilde{M}_{\varepsilon}=\operatorname{dim}_{R} M+1$.

(iii) The boundary of $\widetilde{M}_{\varepsilon}$ contains an open subset $\omega_{1} \subset \omega$ with $p \in \omega_{1}$.

(iv) The tangent cone of $\widetilde{M}_{\varepsilon}$ at $p$ is spanned over $R^{+}$by $T_{p}(M)$ and $J \nu_{\varepsilon} \in$ $N_{p}(M)$.

(v) For each $C^{1}$ CR function $u$ on $\omega$ there is a $C^{1}$ function $U$ on $\widetilde{M}_{\varepsilon}$ with $\left.U\right|_{\omega_{1}}=\left.u\right|_{\omega_{1}}$.

The proof of Theorem 1.4 (given in §3) will require the above theorem and an analysis of the relationship between CR extension to a manifold of one higher 
dimension and the hypoanalytic wave front set of CR functions which we now discuss.

We make use of the mini FBI transform defined in [BRT1]. For $u \in C_{0}^{\infty}(\omega)$, $\omega \subset M$ and $z, \varsigma \in C^{d}, w \in C^{m}$ and $k>0$, the mini FBI transform of $u$ is

$$
\begin{aligned}
F_{k}(u, z, w, \zeta) & :=\int_{x \in R^{d}} u(Z(x, w), w) e^{-i \varsigma \cdot Z(x, w)-k\langle\varsigma\rangle[z-Z(x, w)]^{2}} d_{x} Z(x, w) \\
& =\int_{z^{\prime} \in \omega_{w}} u\left(z^{\prime}, w\right) e^{-i \varsigma \cdot z^{\prime}-k\langle\varsigma\rangle\left[z-z^{\prime}\right]^{2}} d z^{\prime}
\end{aligned}
$$

where $\langle\zeta\rangle=\left(\zeta_{1}^{2}+\cdots+\zeta_{d}^{2}\right)^{1 / 2}$ which makes sense provided $|\operatorname{Re} \varsigma|>|\operatorname{Im} \varsigma|$. This transform is slightly different than the one defined in [BCT] which involves an integral over the $\operatorname{Re}(w)$ variables as well as the $x$ variables. As noted below, many of the results in [BCT] are valid using the mini FBI transform with only minor modifications in their proofs.

In [BCT], they define the concept of the hypoanalytic wave front set of a single function over a point $p \in M$. Since we are interested in the hull of holomorphy of an open set $\omega \subset M$ (with $p \in M$ ) we define the concept of the hypoanalytic wave front set of $\mathrm{CR}(\omega)$ over a point $p \in M$. Our definition differs from the one in [BCT] only in the use of quantifiers. Our point $p$ will be the origin.

DEFINITION 1.6. Let $\omega$ be an open set in $M$ which contains 0 . Fix $g \in C_{0}^{\infty}(\omega)$ with $g \equiv 1$ near 0 . We say that a vector $\xi \in T_{0}^{0}(M)$ is not in the hypoanalytic wave front set of $\mathrm{CR}(\omega)$ over 0 if there exist $k, R>0$ and an open cone $\Gamma_{\xi}$ in $C^{d}$ containing $\xi$ and an open set $W$ in $C^{n}$ containing 0 such that for each distribution $u$ which is CR on $\omega$, there is a constant $C>0$ such that

$$
\left|F^{k}(g u, z, w, \varsigma)\right| \leq C e^{-|s| / R}
$$

for all $(z, w) \in W, \varsigma \in \Gamma_{\xi}$.

We denote the hypoanalytic wave front set of $\mathrm{CR}(\omega)$ by $W F_{h a}^{0}(\mathrm{CR}(\omega)) \subset T_{0}^{0}(M)$. This definition can easily be shown to be independent of the cutoff function $g$. Note that the constants $k, R$ and the cone $\Gamma_{\xi}$ and open set $W$ depend only on $\xi$ (and $\omega)$ but not on the CR function $u$ defined on $\omega$. The constant $C$ on the other hand is allowed to depend on $u$. A key component in the proof of Theorem 1.4 is the following lemma which analyzes the relationship between CR extension to a manifold of one higher dimension and the hypoanalytic wave front set of $\operatorname{CR}(\omega)$.

LEMMA 1.7. Suppose $M$ is a CR generic submanifold of $C^{n}$ presented as in (1.1) so that $p=0 \in M$ and suppose $\omega$ is an open set in $M$ which contains 0 . Suppose that $\nu \in T_{0}^{0}(M)$ is a vector such that there exists a manifold with boundary denoted by $\widetilde{M}$ with the following properties:

(i) $\operatorname{dim}_{R} \widetilde{M}=\operatorname{dim}_{R} M+1$,

(ii) The boundary of $\widetilde{M}$ contains an open set $\omega_{1} \subset \omega$ in $M$ with $0 \in \omega_{1}$,

(iii) The tangent cone of $\widetilde{M}$ at 0 is spanned over $R^{+}$by $J \nu \in N_{0}(M)$ and $T_{0}(M)$,

(iv) For every $C^{1} C R$ function $u$ on $\omega$ there is a $C^{1} C R$ function $U$ on $\widetilde{M}$ with $\left.U\right|_{\omega}=\left.u\right|_{\omega_{1}}$.

Then $W F_{h a}^{0}(\mathrm{CR}(\omega))$ is contained in the closed half space $\Gamma_{\nu}:=\left\{w \in T_{0}(M) ; w\right.$. $\nu \geq 0\}$. 
This result can be thought of as a limiting version of some of the results in [BCT] where it is shown that if CR functions can locally be extended to an open wedge in $C^{n}$ with cross section given by the cone $J(\Gamma)$ (where $\Gamma \subset T_{0}^{0}(M)$ ), then the hypoanalytic wave front sets at 0 of CR functions are contained in the polar of $\Gamma, \Gamma^{o}:=\left\{w \in T_{0}(M) ; w \cdot \nu \geq 0\right.$, for all $\left.\nu \in \Gamma\right\}$. Clearly, as $\Gamma$ shrinks to the ray spanned by the vector $\nu \in \Gamma$, then $\Gamma^{o}$ approaches the half space $\Gamma_{\nu}$. In other words, one would conjecture, based on the results in [BCT] that if one can CR extend in the direction $J \nu$, then the hypoanalytic wave front sets of CR functions should be contained in the half space $\Gamma_{\nu}$. Roughly speaking, the content of Lemma 1.7 is that this conjecture is true.

2. Proof of Lemma 1.7. In view of [BCT, Lemma I.4.1 and equation (2.15)], it suffices to restrict our attention to CR functions of class $C^{1}$. Let $\nu, p, \omega_{1} \subset M$ be given as in Lemma 1.7. Suppose $\xi$ is a vector which is not in $\Gamma_{\nu}$. Fix $g \in C_{0}^{\infty}\left(\omega_{1}\right)$ which is one on a set $\omega_{2}$ where $\omega_{2} \subset \subset \omega_{1}$ and such that $0 \in \omega_{2}$. We must show that there are constants $k, R>0$ and an open cone $\Gamma_{\xi}$ in $C^{d}$ containing $\xi$ and an open set $W$ such that for each $u$ which is CR on $\omega$, there is a constant $C>0$ such that

$$
\left|F^{k}(g u, z, w, \varsigma)\right| \leq C e^{-|\varsigma| / R}
$$

for all $(z, w) \in W$ and $\zeta \in \Gamma_{\xi}$.

Arrange coordinates as in (1.1) so that $p$ is the origin and where $Z(x, w)=$ $x+i \phi(x, w)$ with $\phi(0,0)=0$ and $D \phi(0,0)=0$. Now the tangent cone of $\widetilde{M}$ at $p=0$ is spanned over $R^{+}$by $T_{0}(M)=\left\{(x+i 0, w) ; x \in R^{d}, w \in C^{m}\right\}$ and $J \nu \in N_{0}(M)=\left\{(0+i y, 0) ; y \in R^{d}\right\}$. Therefore we can find $\delta_{1}^{0}>0$ and $\delta_{2}^{0}>0$ and arrange a local parameterization of $\widetilde{M}$ of the form $(x, w, t) \rightarrow(\widetilde{Z}(x, w, t), w)$ for $x \in R^{d}, w \in C^{m}$ with $|x|<\delta_{2}^{0}$ and $|w|<\delta_{2}^{0}$, and $0 \leq t \leq \delta_{1}^{0}$. Here $\widetilde{Z}$ has the form

$$
\widetilde{Z}(x, w, t)=Z(x, w)+i q(x, w, t)
$$

where $q$ is a smooth real valued function with $q(x, w, 0)=0$ and $\partial q(0,0,0) / \partial t=\nu$. Since $\xi$ is not in $\Gamma_{\nu}$ i.e. $(\xi \cdot \nu<0)$ we can find an $\varepsilon>0$ and shrink $\delta_{1}^{0}$ and $\delta_{2}^{0}$ if necessary so that

$$
q(x, w, t) \cdot \xi \leq-\varepsilon t|\xi|
$$

for $0 \leq t \leq \delta_{1}^{0},|x| \leq \delta_{2}^{0}$ and $|w| \leq \delta_{2}^{0}$. Fix $\delta_{1}, \delta_{2}$ with $0<\delta_{1}<\delta_{1}^{0}$ and $0<\delta_{2}<\delta_{2}^{0}$. The constants $\delta_{1}$ and $\delta_{2}$ will be chosen later. Our first requirement on $\delta_{2}$ is that

$$
\left\{(Z(x, w), w) ;|x|<\delta_{2},|w|<\delta_{2}\right\} \subset \omega_{2} .
$$

Choose a function $g_{0} \in C_{0}^{\infty}\left(\left\{x \in R^{d} ;|x|<\delta_{2}\right\}\right)$ with $g_{0}(x)=1$ for $|x| \leq \delta_{2} / 2$. For fixed $w \in C^{m}$ with $|w|<\delta_{2}$ define

$$
\begin{gathered}
\widetilde{M}_{w}:=\left\{\left(\widetilde{Z}\left(x, w, t g_{0}(x)\right), w\right) ;|x|<\delta_{2}, 0 \leq t \leq \delta_{1}\right\} \\
M_{w, \delta_{1}}:=\left\{\left(\widetilde{Z}\left(x, w, g_{0}(x) \delta_{1}\right), w\right) ;|x|<\delta_{2}\right\} \\
M_{w}:=\left\{(Z(x, w), w) ;|x|<\delta_{2}\right\}
\end{gathered}
$$


Note that $\operatorname{dim}_{R} M_{w}=\operatorname{dim}_{R} M_{w, \delta_{1}}=d$ and that $M_{w}$ and $M_{w, \delta_{1}}$ together forms the boundary of $\widetilde{M}_{w}$. Therefore by Stokes' theorem, we may write

$$
\begin{aligned}
F^{k}(g u, z, w, \zeta)= & \int_{z^{*} \in M_{w}} u\left(z^{*}, w\right) g\left(z^{*}, w\right) e^{-i \varsigma \cdot z^{*}-k\langle\varsigma\rangle\left[z-z^{*}\right]^{2}} d z^{*} \\
= & \int_{z^{*} \in M_{w, \delta_{1}}} u\left(z^{*}, w\right) g\left(z^{*}, w\right) e^{-i \zeta \cdot z^{*}-k\langle\varsigma\rangle\left[z-z^{*}\right]^{2}} d z^{*} \\
& +\int_{z^{*} \in \tilde{M}_{w}} d_{z^{*}}\left[U\left(z^{*}, w\right) g\left(z^{*}, w\right) e^{-i \zeta \cdot z^{*}-k\langle\varsigma\rangle\left[z-z^{*}\right]^{2}} d z^{*}\right]
\end{aligned}
$$

Here, we have used the notation $z^{*}=\left(z_{1}^{*}, \ldots, z_{d}^{*}\right) \in C^{d}$ and $d z^{*}=d z_{1}^{*} \wedge \cdots \wedge d z_{d}^{*}$. Also in the second integral, we have denoted the CR extension of $u$ to $\widetilde{M}$ by $U$, which is guaranteed to exist by hypothesis. We have also assumed that $g$ has been extended to $\widetilde{M}$ so that $g \equiv 1$ on $\widetilde{M}_{w}$ (recall that originally $g \equiv 1$ on $\omega_{2}$ ). In view of type considerations, only the $\bar{z}^{*}$ derivatives are applied to the integrand of the second integral. Since $U$ is CR, and $g \equiv 1$ on $\widetilde{M}_{w}$, this integrand vanishes.

Now, we analyze the first integral on the right side of (2.5). Let us denote by $Q\left(\varsigma, z^{*}, z\right)$ the exponent appearing in the integrand. We first analyze $Q$ when $\varsigma=\dot{\xi}=\xi /|\xi|, z=0$ and

$$
z^{*}=\tilde{Z}\left(x, w, g_{0}(x) \delta_{1}\right)=x+i\left(\phi(x, w)+q\left(x, w, g_{0}(x) \delta_{1}\right)\right) \in M_{w, \delta_{1}} .
$$

We obtain

$$
\operatorname{Re}(Q)=\dot{\xi} \cdot \phi(x, w)+\dot{\xi} \cdot q\left(x, w, g_{0}(x) \delta_{1}\right)-k|x|^{2}+k\left(\phi(x, w)+q\left(x, w, g_{0}(x) \delta_{1}\right)\right)^{2}
$$

We separately consider two cases; when $|x| \leq \delta_{2} / 2$; and when $\delta_{2} / 2 \leq|x| \leq \delta_{2}$. In the latter case we obtain

$$
\operatorname{Re}(Q) \leq C\left(\delta_{2}^{2}+|w|^{2}\right)-\frac{k \delta_{2}^{2}}{4}+C k\left(\delta_{2}^{4}+|w|^{4}+\delta_{1}^{2}\right)
$$

where we have used the estimates

$$
\begin{gathered}
\dot{\xi} \cdot q\left(x, w, g_{0}(x) t\right) \leq 0, \quad \text { cf. }(2.2) \\
|\phi(x, w)| \leq C\left(|x|^{2}+|w|^{2}\right) \\
\left|q\left(x, w, g_{0}(x) \delta_{1}\right)\right| \leq C \delta_{1}, \quad \text { recall } q(x, w, t=0)=0
\end{gathered}
$$

where $C$ is a uniform constant which is independent of the parameters $t, x, w, \delta_{1}, \delta_{2}$, and $k$. If we restrict $\delta_{2}$ so that $C \delta_{2}^{4} \leq \delta_{2}^{2} / 8$ and then fix $k \geq 8 C+8$ then we obtain

$$
\begin{aligned}
& \operatorname{Re}\left\{Q\left(\varsigma=\dot{\xi}, z^{*}=\tilde{Z}\left(x, w, g_{0}(x) \delta_{1}\right), z=0\right)\right\} \\
& \quad \leq-\delta_{2}^{2}+C\left(|w|^{2}+k|w|^{4}\right)+C k \delta_{1}^{2} \leq-\delta_{2}^{2} / 2+C\left(|w|^{2}+k|w|^{4}\right)
\end{aligned}
$$

provided we take $\delta_{1}=\delta_{2} / \sqrt{2 C k}$.

In the former case, we have $|x| \leq \delta_{2} / 2$ and so $g_{0}(x)=1$ and $\dot{\xi} \cdot q\left(x, w, \delta_{1}\right)<-\varepsilon \delta_{1}$ (cf. (2.2)). From (2.6), we obtain

$$
\begin{aligned}
\operatorname{Re}\{Q & \left.\left(\varsigma=\dot{\xi}, z^{*}=\widetilde{Z}\left(x, w, g_{0}(x) \delta_{1}\right), z=0\right)\right\} \\
& \leq C\left(\delta_{2}^{2}+|w|^{2}\right)-\varepsilon \delta_{1}+k C\left(\delta_{2}^{4}+|w|^{4}+\delta_{1}^{2}\right) \\
& \leq C\left(\delta_{2}^{2}+|w|^{2}\right)-\varepsilon \delta_{2} / \sqrt{2 C k}+k C\left(\delta_{2}^{4}+|w|^{4}+\delta_{2}^{2} / 2 C k\right)
\end{aligned}
$$

where we have used our choice of $\delta_{1}$ above. 
Note in both of the above cases that $\operatorname{Re} Q\left(\varsigma=\dot{\xi}, z^{*}=\widetilde{Z}\left(x, w, g_{0}(x) \delta_{1}, z=0\right)\right)<$ 0 provided $\delta_{2}$ (and hence $\delta_{1}$ ) is chosen suitably small and provided $w$ is in some suitably small neighborhood of 0 . Since $Q\left(\varsigma, z^{*}, z\right)$ is smooth in all arguments and homogeneous of degree one in $|\zeta|$, we can find an open set $W$ containing 0 and a cone $\Gamma_{\xi}$ containing $\xi$ and constants $c_{0}, \delta_{1}, \delta_{2}>0$ such that with $M_{w, \delta_{1}}$ defined as in $(2.4 \mathrm{~b})$ we obtain

$$
\operatorname{Re}\left[Q\left(\varsigma, z^{*}, z\right)\right] \leq-c_{0}|\zeta|
$$

for $\zeta \in \Gamma_{\zeta}, z^{*} \in M_{w, \delta_{1}}$, and $(z, w) \in W$. This estimate together with the fact that the second integral on the right side of (2.5) vanishes establishes (2.1) with $R=1 / c_{0}$ as desired.

3. The proof of Theorem 1.4. First, we arrange that $p=0$ and assume that $M$ is presented as in (1.1). It suffices to prove the following: if $\Gamma^{\prime}$ is a smaller cone than $\Gamma$, then there exists an open set $\omega^{\prime}$ in $M$ containing $p$ and an open set $V^{\prime}$ in $C^{n}$ containing $p$ such that for each CR distribution $u$ on $\omega$ there is a holomorphic function $U \in B_{V^{\prime}}\left(\omega^{\prime}, \Gamma^{\prime}\right)$ with $b U=u$ on $\omega^{\prime}$. For then one may take $\Omega=\bigcup N_{V^{\prime}}\left(\omega, J\left\{\Gamma^{\prime}\right\}\right)$ where the union is taken over all cones $\Gamma^{\prime}$ which are smaller than $\Gamma$.

To prove the above, we need the following theorem which is essentially the content of Theorem II2.2 in [BCT].

THEOREM 3.1. Let $\omega$ be an open set in $M$ containing 0 . Let $\Gamma$ be a closed acute convex cone in $T_{0}^{0}(M)$ with $W F_{h a}^{0}(\mathrm{CR}(\omega)) \subset \Gamma^{o}:=\left\{\nu \in T_{0}^{0}(M) ; \nu \cdot \xi \geq 0\right.$ for all $\xi \in \Gamma\}$. Suppose $\Gamma^{\prime}$ is a smaller cone than $\Gamma$. Then there is an open set $\omega^{\prime}$ in $M$ containing 0 and an open set $V^{\prime}$ in $C^{n}$ containing 0 such that for each $C R$ distribution $u$ on $\omega$ there is a holomorphic function $U \in B_{V^{\prime}}\left(\omega^{\prime}, \Gamma^{\prime}\right)$ with $b U=u$ on $\omega^{\prime}$.

This theorem is just a mini FBI version of Theorem II.2.2 in [BCT]. As mentioned in $\S 1$, the mini FBI transform defined there uses an integral over $R^{d}$ (the $x$ variables) which is isomorphic to the totally real part of the tangent space of $M$ at 0 , whereas in [BCT], the FBI transform uses an integral over a maximally real subspace of $T_{0}(M)$. Therefore in [BCT], the extension of the CR function is clearly holomorphic in all variables but the conoid in which the holomorphic extension is defined is not the same as the conoid that we are seeking. In our context, it is clear that $F^{k}(u, z, w, \zeta)$ as defined in $\S 1$ is formally holomorphic in $z$, but not necessarily in $w$. Therefore, if one repeats the proof of Theorem II.2.2 in [BCT] using the mini FBI transform and by treating $w$ as a parameter one obtains the conoid $N_{V^{\prime}}\left(\omega^{\prime}, \Gamma^{\prime}\right)$ as described above and one obtains that any function $u$ which satisfies (2.1) for $\zeta=\xi$ not in $\Gamma^{o}$ is the distributional boundary values on $\omega^{\prime}$ of a function $U$ such that $U(z, w)$ is holomorphic in $z$ whenever $(z, w) \in N_{V^{\prime}}\left(\omega^{\prime}, \Gamma^{\prime}\right)$. The quantifiers in Definition 1.6 are arranged so that the open set $N_{V^{\prime}}\left(\omega^{\prime}, \Gamma^{\prime}\right)$ does not depend on $u$.

When $u$ is CR, then we can show that $U(z, w)$ is holomorphic in $w$ as well as $z$ as follows. Let $X_{1}, \ldots, X_{m}$ be a basis for $H^{0,1}(M)$ near $p=0$ where each $X_{j}$ has the form

$$
X_{j}=\frac{\partial}{\partial \bar{w}_{j}}+\sum_{k=1}^{d} \alpha_{j k} \frac{\partial}{\partial \bar{z}_{k}}
$$


where the $\alpha_{j k}$ are smooth functions on $M$. We extend the functions $\alpha_{j k}$ so that they are defined on $C^{n}$ and thus we may consider $X_{j}$ as vector fields defined on $C^{n}$. Now since $U$ is holomorphic in $z$, for $(z, w) \in N_{V^{\prime}}\left(\omega^{\prime}, \Gamma^{\prime}\right)$, we have $\partial U / \partial \bar{w}_{j}=X_{j} U$ on $N_{V^{\prime}}\left(\omega^{\prime}, \Gamma^{\prime}\right)$. Moreover in the sense of distribution theory,

$$
b\left(\frac{\partial U}{\partial \bar{w}_{j}}\right)=b\left(X_{j} U\right)=X_{j} u=0
$$

on $\omega^{\prime}$ since $u$ is CR. Now fix an arbitrary $w$ with $(Z(0, w), w) \in \omega^{\prime}$. Let $N_{w}=$ $\left\{z \in C^{d} ;(z, w) \in N_{V^{\prime}}\left(\omega^{\prime}, \Gamma^{\prime}\right)\right\}$. Note that $\omega_{w}^{\prime}:=\left\{z \in C^{d} ;(z, w) \in \omega^{\prime}\right\}$ is a totally real, $d$-dimensional manifold which lies in the boundary of (in fact is the corner of) the complex $d$-dimensional conoid $N_{w}$. Since $b_{w}\left(\partial U / \partial \bar{w}_{j}\right)$ vanishes on $\omega_{w}^{\prime}$ and since $\partial U / \partial \bar{w}_{j}$ is holomorphic on $N_{w}$, clearly $\partial U / \partial \bar{w}_{j}$ vanishes on $N_{w}$. Since $w$ was arbitrarily chosen, clearly $\partial U / \partial \bar{w}_{j} \equiv 0$ on all of $N_{V^{\prime}}\left(\omega^{\prime}, \Gamma^{\prime}\right)$, as desired.

THE REST OF THE PROOF OF THEOREM 1.3. Let $\Gamma$ be the convex hull of the $l$ th Levi form at $p=0$. In view of Theorem 3.1, we wish to show $W F_{h a}^{0}(\mathrm{CR}(\omega)) \subset$ $\Gamma^{o}$. Since the interior of $\Gamma$ (in $R^{d} \approx T_{0}^{0}(M)$ ) is nonempty, $\Gamma^{0}$ is an acute cone. It suffices to show that $W F_{h a}^{0}(\mathrm{CR}(\omega)) \subset \Gamma_{1}^{o}$ for every closed convex cone with $\Gamma_{1}<\Gamma$. Now there exists a cone $\Gamma_{2}$ and unit vectors $\nu_{1}, \ldots, \nu_{N}$ which lie in the image of the $l$ th Levi form such that $\Gamma_{2}$ is the convex hull of $\nu_{1}, \ldots, \nu_{N}$ and $\Gamma_{1}<\Gamma_{2}<\Gamma$. Since each $\nu_{j}$ is in the image of $L_{0}^{l}$, we may use Theorem 1.5 to show that for each $j$ and for each $\varepsilon>0$ there is a unit vector $\nu_{j}^{\varepsilon} \in T_{0}^{0}(M)$ with $\left\|\nu_{j}^{\varepsilon}-\nu_{j}\right\|<\varepsilon$ and which satisfies the hypothesis of Lemma 1.7. Therefore by Lemma 1.7,

$$
W F_{h a}^{0}(\mathrm{CR}(\omega)) \subset \bigcap_{j=1}^{N} \Gamma_{\nu_{j}^{\varepsilon}}=\bigcap_{j=1}^{N}\left\{w \in T_{0}^{0}(M) ; w \cdot \nu_{j}^{\varepsilon} \geq 0\right\}
$$

Since $\left\|\nu_{j}-\nu_{j}^{\varepsilon}\right\| \rightarrow 0$ as $\varepsilon \rightarrow 0$, clearly

$$
\begin{aligned}
W F_{h a}^{0}(\mathrm{CR}(\omega)) \subset \bigcap_{j=1}^{N} \Gamma_{\nu_{j}}=\left\{w \in T_{0}^{0}(M) ; w \cdot \nu_{j} \geq 0\right\} \\
=\Gamma_{2}^{o} \subset \Gamma_{1}^{o} \text { as desired. } \\
\text { BIBLIOGRAPHY }
\end{aligned}
$$

\section{BIBLIOGRAPHY}

[BCT] M. S. Baouendi, C. H. Chang, and F. Treves, Microlocal hypo-analyticity and extension of CR functions, J. Differential Geom. 18 (1983), 331-391.

[BRT1] M. S. Baouendi, L. P. Rothschild, and F. Treves, CR structures with group action and extendability of CR functions, Invent. Math. 82 (1985), 359-396.

[BR] M. S. Baouendi and L. P. Rothschild, Normal forms for generic manifolds and holomorphic extension of CR functions, J. Differential Geom. 25 (1987), 431-467.

[B] A. Boggess, CR extendability near a point where the first Levi form vanishes, Duke Math. J. 48 (1981), 665-684.

[BPi] A. Boggess and J. T. Pitts, CR extension near a point of higher type, Duke Math J. 52 (1985), 67-102.

[BP] A. Boggess and J. C. Polking, Holomorphic extension of CR functions, Duke Math. J. 49 (1982), 757-784.

Department of Mathematics, Texas A \& M University, College Station, TEXAS 77843 